\title{
Contra la violencia: un nuevo discurso para una nueva práctica
}

Lorenzo Romeo*

Resumen. - El cambio de época significa un cambio hacia una nueva fenomenología de la violencia. Si antes toda la sociedad trataba de exorcizar su propia violencia interna proyectando hacia el exterior la imagen del "otro" como enemigo, hoy, paradójicamente, la época de paz replantea la violencia interna: la nueva fenomenología de la violencia se multiplica y se desplaza sobre el diferente, el débil, sobre la misma naturaleza. Por esto son muy importantes los aportes que hoy provienen desde dentro del problema de la violencia; la reflexión de los movimientos feministas, juveniles, indígenas que retoman la ética en el nudo esencial de la relación entre yo y el otro para sentar las bases del proyecto moral de la sociedad que se quiere construir.

\section{Introducción}

La violencia, según su acepción más común, se define como una forma extrema de agresión, como una acción conscientemente dirigida a dañar o disminuir física o psíquicamente a otros. A menudo, la violencia se relaciona con el acto de agresión, olvidando que la agresión no es sólo un acto: es la disposición por parte de un individuo o grupo a actuar de manera que dañe los intereses de otros.

La explicación de las causales sociológicas de esta disposición es muy importante, pero no es suficiente para comprender el cómo y el por qué una actitud agresiva se convierte en acto destructivo. Es decir, un acto de violencia que puede o no (dependiendo de la sociedad) ser considerado un crimen. En la identificación de tales causales, hay que considerar que la violencia, como forma de condicionamiento y de control, se expresa no sólo imponiendo una conducta, sino impidiendo que otro pueda realizar sus potencialidades como

* Departamento de Ciencias Sociales -UCA. persona. En este sentido propiamente sociológico, se recurre al concepto de violencia estructural, una violencia cuyas raíces se encuentran en la desigualdad social y en las relaciones de dominación que se generan a partir de esta desigualdad. En efecto, las relaciones sociales de desigualdad (de género, etáreas, étnica, de clase) son relaciones que estructuralmente implican violencia porque se basan sobre condiciones y posiciones de desigualdad que justifican esta disposición a someter al otro, disposición que en determinado momento se puede convertir en un acto concreto de violencia.

Dentro de esta perspectiva, hay sectores -en particular jóvenes, mujeres y niños/as- cuya situación los expone estructuralmente a vivir de manera más directa experiencias de violencia. La situación de estos sectores se expresa en "circuitos de daño psicosocial". Estos incluyen procesos de deterioro personal en diferentes instancias sociales como el trabajo, la familia, la ciudadanía. También condicionamientos sociales que resultan de la pertenencia a un 
medio pobre y que conllevan una valorización social negativa con su consiguiente estigmatización.

En nuestras sociedades, estos circuitos de daño psicosocial son producto de situaciones ligadas a cambios tanto en el nivel estructural de la sociedad (trabajo, educación, familia) como en el nivel del imaginario social (estigmas, representaciones, creencias, valores).

Con respecto al contexto social, la crisis que estalló en la última década ha dejado como saldo un aumento cuantitativo de la pobreza y el empobrecimiento cualitativo de las condiciones de vida de amplios sectores. Si a la crisis le sumamos los efectos del ajuste, entonces tenemos cambios estructurales que se manifiestan en la caída de los ingresos de importantes sectores de la población y en cambios en el empleo, o sea, una creciente precarización y desempleo.

Estos indicadores en su conjunto nos señalan que estamos ante una redefinición de la sociedad y de las relaciones de poder que apuntan no sólo a un aumento de la desigualdad social, y por lo tanto de la violencia estructural, sino también a una exclusión permanente de los sectores más empobrecidos por parte de este nuevo modelo de sociedad. Estas modificaciones están asociadas a un clima cultural que se manifiesta en desesperanza, ausencia de expectativas favorables, pérdida de ideas futuras y desconfianza en los mecanismos institucionales. Estamos ante un nuevo contexto estructural que imprime un nuevo sello a las relaciones de violencia.

\section{Discurso cultural y orden social}

Las condiciones estructurales no generan automáticamente relaciones de violencia: éstas se construyen siempre a través de discursos culturales. El discur- so no sólo "dice", sino que ordena: nos dice cómo, de qué manera, con qué sentido hablar de cierto tema. Por ejemplo, dependiendo del discurso formularemos unas u otras preguntas sobre el tema de la violencia.

No es lo mismo hablar de violencia a partir de un discurso que nos permita comprender y asumir el problema, que hablar de violencia a partir de un discurso que más bien nos lleve a reproducir relaciones de violencia. Por ejemplo, si nuestro discurso se fundamenta en el miedo ante la amenaza del "otro", cualquier explicación de la violencia del "otro" servirá en realidad para justificar nuestra propia violencia.

Es a través de la práctica discursiva que damos significado a nuestras acciones. De esta manera, el discurso permite controlar la conducta social. Las estructuras sociales se reproducen a partir de cierto control social (que se institucionaliza de diferentes formas). $\mathrm{Y}$ este control es posible a través de cierto discurso cultural, desde el cual se construyen las representaciones de la violencia legítima o no legítima, de lo que es desviación y delito y de cómo la sociedad debe ejercer el control social hasta apelar a formas extremas, como la pena de muerte. El discurso es también creador del orden social, construido por los sujetos y actores sociales. Pero el discurso se construye y opera como creador de orden social sólo en la medida en que se hace "público" y se convierte en la base valorativa y normativa a partir del cual la gente se reconoce como miembro de una comunidad que comparte un proyecto.

Históricamente, este espacio público ha sido representado por el Estado, y en parte, por los partidos políticos, aunque en la práctica histórica de América Latina el Estado ha sido más bien la 
expresión de intereses privados. Pero aun así, el Estado ha tenido cierta función simbólica de referencia a algún proyecto histórico común.

Hoy, ante los procesos de globalización y la crisis de los proyectos nacionalesestatales de desarrollo social, se nos presenta, de una forma realmente inédita, el reto de construir un espacio público a partir de un nuevo contenido éticopolítico que nos permita promover discursos liberatorios en contra de los discursos de dominación y sometimiento.

\section{Sociedad civil y construcción del espacio público}

Dentro de las condiciones necesarias para la construcción de un nuevo espacio público, la sociedad civil cobra un peso fundamental. El desarrollo de la sociedad civil permite trascender el ámbito meramente privado individual y convertir los intereses sociales privados organizados en intereses colectivos públicos. La gobernabilidad democrática, en consecuencia, no depende sólo del Estado, sino también de la manera en que la sociedad civil organiza sus intereses particulares, cómo es capaz de dotarlos de sentido de comunidad y cómo los traslada con eficiencia, a través de los partidos y las organizaciones sociales, a los niveles públicos y políticos.

Esta transformación de los intereses privados en públicos, es una condición imprescindible para la construcción de un orden social democrático, es una tarea que no depende exclusivamente del Estado y los partidos políticos, sino también del desarrollo de las organizaciones sociales y de la existencia de una esfera pública en la que los asuntos de interés colectivo puedan discutirse. En este sentido, la sociedad civil alude a la integración y articulación de las fuerzas sociales por oposición a la sociedad mercantil y fragmentada, propiciando la consolidación del proceso democrático y la ampliación de la ciudadanía, dos ejes básicos para poder abordar los problemas de la inequidad, la discriminación y la exclusión social, factores estructurales que agudizan de forma dramática la violencia de nuestros países.

Pensamos en la reorganización de la sociedad civil en función del surgimiento de formas renovadas de acción colectiva que incorporan nuevos puntos de vista e intereses, desarrollando redes de solidaridad que contrarrestan las fuerzas que tienden a desintegrar el tejido social.

\section{Naturaleza ético-comunicativa de la sociedad civil}

La sociedad civil puede desempeñar este papel de integración social y creación de un nuevo espacio público sólo si consideramos su naturaleza ético-racional o ético-comunicativa. Tal comunicación se deriva de la necesidad no sólo de conocer, sino de evaluar y comprender al otro, del tránsito hacia él a través del habla.

La sociedad civil es por lo tanto expresión de un discurso ético-racional que tiene como fundamento la comprensión del yo hacia el otro, o sea representa la expresión más autentica de la comunicación humana. La ética en el discurso de la sociedad civil radica en descubrir al otro como un ser de respeto. En esta visión, el otro no puede ser tomado, de ninguna manera, como un enemigo a quien hay que destruir. Por el contrario, el otro es común al yo, lo enriquece, lo universaliza y le permite hacer vida comunitaria, vale decir, una vida ética. 
Desde esta perspectiva, la sociedad civil es fundamentalmente un espacio éticocomunicativo de construcción de solidaridad y de búsqueda de nuevos modelos de relación social que propicien la participación de todos los sectores de la sociedad, incluyendo el mismo Estado.

Es de esperar, según este enfoque, que esto contribuya a una nueva institucionalidad en las relaciones entre Estado, mercado y sociedad civil, que haga viable la sostenibilidad de una estrategia de desarrollo con equidad y en función del ser humano. Esto implica avanzar en un proyecto de modernización democrática incluyente, que redefina las relaciones Estado-sociedad.

\section{Modernización excluyente y violencia}

La búsqueda de un nuevo proyecto viable e incluyente de sociedad representa, por sí misma, la base más firme en contra de la violencia que deriva de la imposición de un modelo único de modernización forzada al servicio de las necesidades de una economía mundial transnacionalizada; un modelo que reduce el ser humano a homo oeconomicus, elevando el mercado a expresión universal de la naturaleza humana que justifica éticamente la eliminación de todo aquello que obstaculice la ampliación ilimitada de su lógica. La ausencia de proyectos alternativos refuerza la naturaleza agresiva del mercado mundial como lugar de subordinación y sometimiento de todas las demás culturas del planeta, a las exigencia de un patrón cultural occidental homogeneizante como condición de supervivencia en el mercado.

Sin embargo, la construcción de alternativas de desarrollo exige una transformación teórico-práctica de las mismas nociones de desarrollo y modernidad. Esta transformación se logra, idealmente, partiendo de la práctica de los movimientos sociales, que en nuestros países, se han desarrollado como respuesta a los órdenes hegemónicos resultantes de las ideologías del desarrollo impuestas desde la posguerra. $\mathrm{La}$ comprensión de estas prácticas es esencial para la construcción de visiones alternativas del desarrollo y de la democracia. A la vez, representa una garantía ante el peligro de operaciones de reingeniería social que no cuentan con las bases cognoscitivas y éticas adecuadas.

Los proyectos de desarrollo transcurren al margen de la experiencia del pueblo y se necesitan, por tanto, nuevos tipos de conocimiento que no produzcan esta violenta abstracción. Hablamos de las formas de conocimiento que existen en amplios sectores, como en el caso de las mujeres y los pueblos indígenas, quienes han mantenido prácticas de conocimiento distintas de las de la modernidad. La búsqueda de nuevos proyectos de desarrollo debe basarse en el encuentro entre las formas modernas y populares de conocimiento.

Aprender a ver esos movimientos no como el reflejo de la crisis, sino como producto de la propia racionalidad y organización que ellos mismos producen, representa un enorme reto teórico, epistemológico y metodológico. Saber enfrentar este reto nos permitirá entrever distintas opciones y hacer visibles otros proyectos y otras culturas, abriendo la reflexión hacia la construcción de otros paradigmas que nos permitan recobrar la esperanza para volver a mirar al futuro, demostrando que no existe un camino único, un camino que, por lo demás, no es viable para la mayoría de la población mundial. 
Ante las relaciones de violencia que derivan de los procesos acelerados de modernización excluyente, las únicas formas eficaces de control social son aquellas que están vinculadas a los procesos de transformación social.

El único discurso que permite controlar la violencia es aquel que permite desarrollar nuevas prácticas de transformación social. Una vez más, los portadores privilegiados de estas nuevas prácticas son los movimientos sociales. Ellos deben convertirse en nuestros principales interlocutores para abordar, comprender y tratar de superar las manifestaciones más inquietantes de la violencia que hoy vivimos.

\section{Control social y transformación}

Hablando de las manifestaciones más inquietantes de la violencia actual de nuestros países, me referiré a la violencia doméstica para demostrar como el movimiento de mujeres ha logrado proponer una nueva forma de control social que va más allá de la mera represión y se ejerce en función de la transformación del discurso y de las relaciones sociales que generan las prácticas de violencia.

Desde mediados de los años 70 , la lucha contra la violencia sexual ha sido el eje articulador del movimiento de las mujeres en América Latina. Esto fue lo que causó que el Estado se hiciera interlocutor del movimiento. La visión de género permitió profundizar y entender que hay una violencia social muy amplia, de la que deriva la violencia contra la mujer y que la misma construcción jerarquizada de la sociedad, al dar preeminencia a todo lo masculino, es una forma de violencia.

La asignación de tareas en función del sexo, la desigualdad construida a partir de la diferencia, la desvalorización de lo femenino, son formas de violencia contra la mujer. La violencia por tanto se concibe no solamente como el acto del hombre que le pega a la mujer, sino como un problema de relación entre sexos que son construidos socialmente. $Y$ en esta construcción la violencia juega un papel importante, que trasciende incluso a la misma mujer como "objeto privilegiado".

La violencia que deriva de cierto discurso sobre masculinidad es una violencia de hombres contra sí mismos, contra otros hombres y contra otras mujeres. Si nosotros construimos relaciones sociales donde la violencia se interpreta como demostración de fuerza y la fuerza es un atributo masculino, ya estamos reproduciendo el estereotipo del violador y minimizando el peso de las relaciones sociales y políticas entre los géneros.

La violencia sexual es el punto culminante de ciertos patrones, construidos y aceptados socialmente, que incluyen entre los atributos masculinos cierta disposición agresiva. Antes de ser un "acto", la violencia sexual es un supuesto de la relación desigual entre los géneros, previo a las relaciones que establecen los individuos particulares. Es un supuesto que implica la apropiación y el daño, la cosificación de las mujeres, el ultraje a su intimidad y a su integridad como persona. Es un supuesto que se basa en un discurso cultural. ¿Por qué en estas últimas décadas la violencia contra las mujeres se ha convertido en un problema social y en algunos casos incluso en crimen?

Ha sido posible precisamente porque el movimiento de las mujeres ha generado un contradiscurso, desarticulando el discurso dominante que produce relaciones desiguales de género y crea formas de control social para seguir reproduciéndolas, para articular otro discurso que 
permita hacer visible la violencia sexual como hecho social y cultural que implica la degradación de la persona, de su autodeterminación. Por lo tanto, no es sólo un problema de las mujeres sino de todos.

El nuevo discurso y las nuevas prácticas de los movimientos de mujeres, paulatinamente hicieron posible la creación de un nuevo espacio público y la ampliación del que ya existía.

Este espacio público, según reconoce el Programa de Desarrollo de las Naciones Unidas (PNUD), puede permitir una nueva forma de control social, o sea, institucionalizar nuevas formas de control social, un control dirigido no solamente a castigar al que maltrata, sino a transformar las relaciones entre géneros como premisa para que termine el maltrato.

El control social, actúa así no como una forma de restricción de la ciudadanía, sino más bien de su ampliación, permitiendo no sólo proteger los derechos humanos, sino también transformar las relaciones sociales que impiden su libre expresión.

\section{Superar la lógica de la dominación}

Los derechos humanos son principios cuyo poder transformador funciona sólo si éstos se integran a otros procesos de transformación de los códigos simbólicos del imaginario social; esos principios tienen poder transformador siempre a través de cierto discurso cultural.

No hay que olvidar que estos códigos simbólicos que ordenan nuestro discurso y por lo tanto la manera de abordar los temas, tienen raíces muy profundas que radican en nuestra propia civilización.
En la civilización occidental, por ejemplo, hay una larga tradición de pesimismo sobre la naturaleza humana considerada como violenta y compulsiva. A partir de esta premisa, el corolario será la necesidad de un orden externo que norme al ser humano desde fuera para proteger la comunidad de sus propios integrantes.

Estas concepciones filosóficas sobre el carácter violento de la humanidad tienen raíces en una larga práctica histórica que subyace a la construcción de la civilización moderna. Detras de esta concepción pesimista que considera la violencia como inherente al ser humano, hay un verdadero miedo ante la amenaza del otro que refleja la experiencia de siglos de una historia donde para afirmarse como sujetos se ha tenido que recurrir a la violencia. En el caso de la civilización occidental, la constitución de la subjetividad y de los sujetos ha seguido la misma evolución del pensamiento moderno, o sea una lógica de oposiciones binarias: blanco/negro; hombre/mujer; mestizo/indio. La base de esta lógica es la misma dominación, una lógica donde el otro se somete o se aniquila.

Esta forma pesimista de abordar el problema del vínculo social, el problema básico del yo y del otro, radica en una profunda contradicción de nuestra civilización. Una contradicción no resuelta, violenta y suicida. Porque si es cierto que la identidad personal o social no puede formarse sin reconocimiento de la diferencia, sin embargo, a lo largo de la civilización occidental la diferencia ha sido considerada obscuramente como un peligro externo. Se trata, como decíamos, de una contradicción violenta y suicida, porque si es cierto que la identidad se construye diferenciándose del otro, cuando el otro se aniquila, la identidad se disuelve también. 
Al proteger mi identidad absolutizándola al extremo -la forma actual de individualismo-, acabo por suprimirme a mí mismo: para salvarme, muero. Esta es la razón por la que hoy ciertos temas de los movimientos indígenas y feministas son centrales, pues tocan el nudo del problema: el problema no resuelto de la relación entre yo y el otro.

\section{Una nueva \\ fenomenología de la violencia}

¿Qué tiene que ver esta reflexión con la violencia de hoy? La violencia de hoy es la violencia cotidiana y difusa, no es la violencia del ángel exterminador o del vengador justiciero, es una violencia sin rostro, anónima y sin objetivo, está en el aire como la contaminación.

El cambio de época significa también un cambio hacia una nueva fenomenología de la violencia. Si antes toda la sociedad trataba de exorcizar su propia violencia interna proyectando hacia el exterior la imagen del "otro" como enemigo, hoy, paradójicamente, la época de paz replantea la violencia interna. Se diluyen las fronteras, pero la guerra se convierte en enfrentamiento de todos contra todos.

Esto es particularmente peligroso en sociedades como las nuestras, donde la legitimidad que se generó a partir de los procesos de paz y de las elecciones todavía no ha logrado consolidarse en un clima estable de institucionalidad democrática. La violencia se desata así sin mediaciones, sin objetivos y parece retornar a una primitiva ansia de matar y muchas veces a quien matamos es al que está más cerca: la nueva fenomenología de la violencia se multiplica y se desplaza sobre el diferente, el débil, sobre la misma naturaleza.

Si nosotros creemos en la posibilidad de una cultura de no violencia, ésta debe transitar por la construcción de un nuevo discurso que debe medirse con las raíces de la violencia. Si en el centro de este discurso está el problema de la relación entre yo y el otro, entonces nosotros no podemos considerarnos como simples observadores o analistas externos.

\section{Bibliografía}

-HERDÅNDEZ, R. (1975). La idea de sociedad civil. México, UNAM.

-BARCELONA, P. (1992). Postmodernidad y comunidad. El regreso de la vinculación total. Madrid.

Trota S.A.

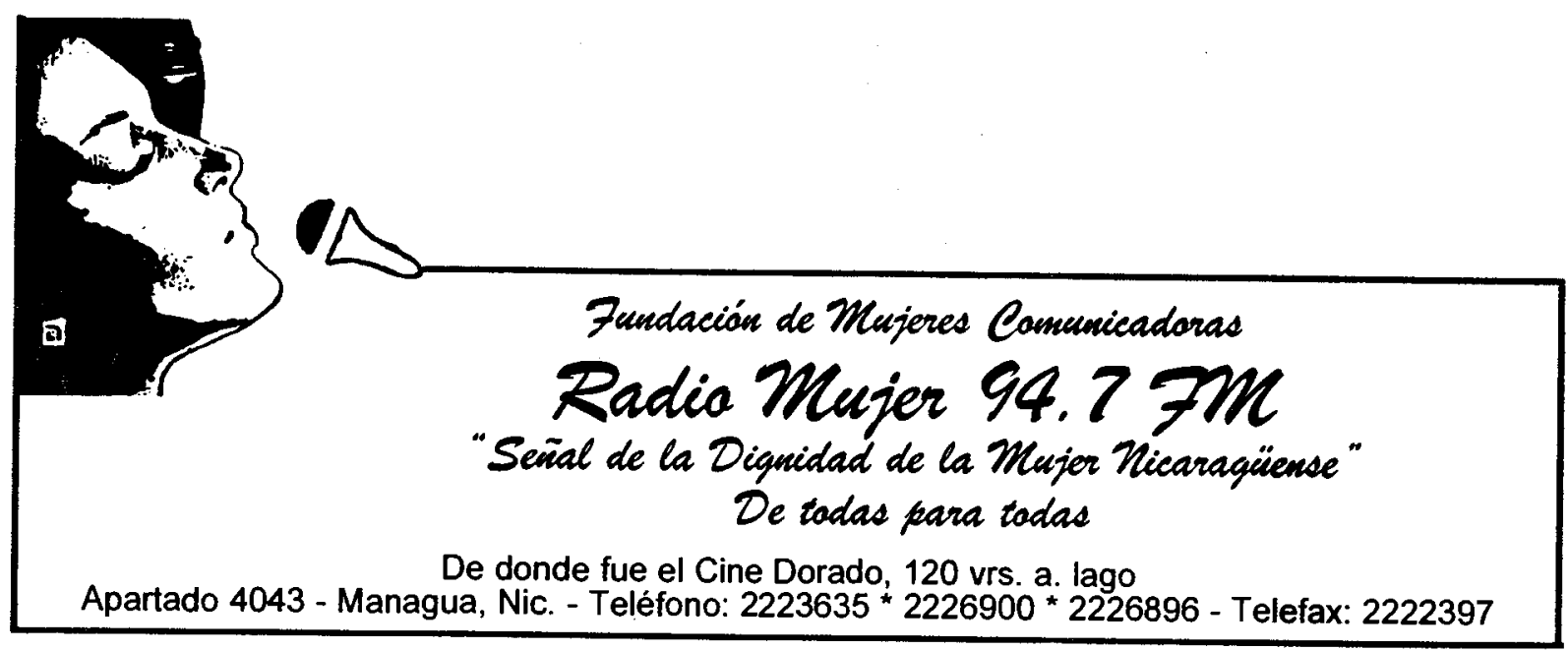

